Annuaire suisse de politique de développement

$19 \mid 2000$

Pour une politique plus cohérente envers les pays en développement

\title{
3. Environnement et développement
}

Catherine Schümperli Younossian et Emilie Mottier

\section{(2) OpenEdition}

Journals

Édition électronique

URL : http://journals.openedition.org/aspd/858

DOI : 10.4000/aspd.858

ISSN : 1663-9669

Éditeur

Institut de hautes études internationales et du développement

Édition imprimée

Date de publication : 1 avril 2000

Pagination : $177-200$

ISSN : 1660-5934

\section{Référence électronique}

Catherine Schümperli Younossian et Emilie Mottier, «3. Environnement et développement », Annuaire suisse de politique de développement [En ligne], 19 | 2000, mis en ligne le 19 août 2012, consulté le 07 septembre 2020. URL : http://journals.openedition.org/aspd/858 ; DOI : https://doi.org/10.4000/aspd. 858 


\section{ENVIRONNEMENT ET DÉVELOPPEMENT*}

\subsection{SUIVI DE LA CONFÉRENCE DE RIO}

Lors de la Conférence des Nations Unies pour le développement et l'environnement, en 1992 à Rio, la communauté internationale s'est engagée à concevoir et à mettre en ouvre une politique visant à favoriser le développement durable. Chaque année se tient à New York la Commission pour le développement durable $(C D D)$, chargée de contrôler et d'encourager sa mise en application. La Suisse a également concrétisé son engagement par l'adoption d'une stratégie pour le développement durable publiée par le Conseil fédéral en 1997. En 1999, la Confédération a notamment mené une campagne de sensibilisation envers les communes, les encourageant à adopter des agendas 21 locaux; elle a également travaillé à la définition d'indicateurs de développement durable. En outre, le peuple suisse a adopté en votation populaire, en avril 1999, la nouvelle Constitution fédérale qui fait mention explicite du développement durable dans son préambule et ses buts.

\section{Commission pour le développement durable (CDD-7)}

La Commission pour le développement durable a été créée à la suite de la Conférence de Rio pour contrôler et encourager la mise en œuvre des chapitres de l'Agenda 21. Depuis 1993, elle se réunit chaque année afin de discuter de cette mise en œuvre et de donner des recommandations pour les travaux ultérieurs au niveau national et international. La CDD est une commission du Conseil économique et social (ECOSOC) de l'ONU; elle compte 53 pays membres qui sont élus pour une période de trois ans selon une clé de répartition géographique. En vertu de ce principe de rotation, la Suisse, qui était membre de ce conseil depuis 1996, y a participé jusqu' au 30 avril 1999.

La Commission pour le développement durable s'est réunie pour la septième fois (CDD-7) à New York, du 19 au 30 avril 1999) ${ }^{1}$. La délégation suisse, conduite par le conseiller fédéral Flavio Cotti, était composée de représentants de l'OFEFP, de la DDC et du Seco. Les ONG étaient représentées par la Communauté de travail.

Les participants ont travaillé dans différents comités de rédaction chargés de négocier des projets de décisions, approuvés ensuite en séance plénière. Cette septième session était consacrée à la promotion d'un tourisme durable, aux modèles de consommation et de production, aux mers et océans, ainsi qu'au «programme d'action de la Barbade en faveur des petits Etats insulaires». Deux journées et demie de la session ont été consacrées au segment ministériel, durant lequel 89 ministres ou officiels de haut rang ont présenté des déclarations nationales. Durant son allocution, le conseiller fédéral Cotti a rappelé que «les pays en développe-

Parties 3.1, 3.2, 3.3: par Catherine Schümperli Younossian, chargée de recherche à l'IUED. Partie 3.4: par Emilie Mottier, diplômée IUED.

1. Le résumé complet ainsi que les principales décisions de la CDD-7 se trouvent sur le site du Bulletin des négociations de la Terre à la page www.iisd.ca/vol05/enb05132f.html 
ment ont besoin d'une forte croissance pour pouvoir satisfaire les besoins essentiels de leurs populations, qu'il s'agisse de nourriture, de soins, de logement ou d'éducation. Cependant, cette croissance ne se traduira en une amélioration des conditions de vie qu'à la condition d'être basée sur des modes de production et de consommation compatibles avec le développement durable. Pour arriver à ce résultat, il faudra un engagement déterminé de tous les pays. Les pays industrialisés ont là une responsabilité particulière; ils doivent améliorer l'efficience de leurs modes de production et réduire leur part, aujourd'hui largement disproportionnée, dans l'utilisation des ressources limitées de la planète».

Position de la Suisse

La Suisse a principalement concentré ses interventions sur le tourisme et les modes de production et de consommation. Dans le domaine du tourisme, elle s'est engagée en faveur de conditions de travail humaines et dans la lutte contre la prostitution enfantine. Avec le soutien de l'Union européenne, elle a insisté pour que la problématique de l'exploitation sexuelle soit prise en compte. Pour illustrer cette problématique, la Suisse a présenté les résultats d'une étude sur la prostitution enfantine, Quick Money - Easy Money ${ }^{2}$. En tant que pays alpin, elle a également veillé à ce que le point de vue des Etats concernés par le tourisme alpin soit pris en considération dans les documents. Pour développer sa position, elle a édité une brochure, Tourism and Sustainable Mountain Development ${ }^{3}$.

S'agissant des modèles de consommation et de production, la délégation suisse a fait part de ses préoccupations, à savoir l'utilisation d'instruments économiques, la réforme fiscale écologique, la suppression de subventions dommageables pour l'environnement et la promotion d'écolabels. A cette occasion, elle a présenté une brochure ${ }^{4}$ qui décrit très concrètement une dizaine d'initiatives privées permettant de rendre les habitudes de consommation et de production plus durables. La gamme des exemples retenus est très large, des placements financiers verts à la politique des transports, en passant par la production et la distribution de produits agricoles biologiques. Mais l'Etat a également un rôle important à jouer dans la définition de conditions-cadres encourageant les modes de production durable (taxes d'incitation ou instruments de financement). Pour chacun des sujets abordés, la brochure dresse un état des lieux des mesures étatiques suisses.

\section{Suivi de Rio en Suisse}

\section{Les agendas 21 locaux}

La mise en œuvre de l'Agenda 21, que la Suisse a approuvé à Rio en juin 1992, ne concerne pas seulement la communauté internationale et les gouvernements nationaux, mais nécessite également l'appui des pouvoirs locaux. Le chapitre 28 de l'Agenda 21 est précisément consacré aux «Initiatives des collectivités publiques à l'appui de l'Agenda 21» et son objectif essentiel consiste à demander à toutes les communes d'établir un agenda 21 local avant 1996. Si l'Agenda 21 adopté à Rio est un instrument de référence et standardisé, les agendas 21 locaux doivent, au contraire, être spécifiques aux réalités politiques, économiques,

2. Christine Plüss, Quick Money - Easy Money: A Report on Child Labour in Tourism, DDC, Bern, June 1999.

3. Center for Development and Environment (CDE), Institute of Geography, University of Bern, Mountains of the World: Tourism and Sustainable Development, CDE-SDC, Bern, 1999.

4. OFEFP, La production durable, une réalité. 10 exemples en provenance de Suisse, OFEFP, Berne, 1999. 
sociales, géopolitiques, institutionnelles de chaque communauté. Dans le cadre du processus d'élaboration d'un agenda 21 local, la concertation entre les différents acteurs de la société est un élément essentiel (autorités communales et administratives, population, organisations non gouvernementales et économie locale). C'est dans le cadre de forums d'échanges et de discussions que se concrétise la stratégie d'un développement durable pour une ville ou une région.

Depuis l'été 1997, l'Office fédéral des forêts, de l'environnement et du paysage (OFEFP) a lancé, à l'attention des communes suisses, un programme d'encouragement aux projets de développement durable au niveau local. La Confédération accorde une contribution financière de 50'000 francs aux projets retenus dans le cadre du programme. Dans le courant de l'automne 1999, on dénombrait une quinzaine de communes ayant bénéficié de ce soutien. Depuis le lancement de ce programme, l'intérêt pour la mise en œuvre du développement durable au niveau local ne cesse de croître et de très nombreux séminaires ont eu lieu au courant de l'année 1999 sur ce thème. Le 18 novembre 1999, à l'invitation de l'OFEFP, s'est tenue la première conférence annuelle consacrée aux agendas 21 locaux.

Afin de faciliter l'échange d'expériences sur les procédures d'agendas locaux, l'Office de l'environnement a ouvert un site Internet ${ }^{5}$, sur lequel on trouve des exemples de communes engagées dans un processus d'agenda 21 local, mais également le programme des manifestations sur le thème ainsi qu'une liste exhaustive de documentation et de liens Internet. Pour sensibiliser les communes, l'OFEFP a publié une brochure à leur intention ${ }^{6}$. Ce document donne une vue d'ensemble de la problématique; il ne fournit pas de solutions toutes faites mais, passant en revue l'Agenda 21, suggère des mesures pouvant être mises en œuvre au niveau communal.

Certains cantons se sont également engagés sur la voie du développement durable en incitant un processus à long terme, à l'exemple du canton du Valais, qui, dans l'établissement de son dossier de candidature pour l'obtention des Jeux olympiques en 2006, a créé un département du développement durable 7 . Sion n'a pas obtenu les Jeux, mais la Fondation pour le développement durable des régions de montagne créée à cette occasion va poursuivre son travail. A la demande du gouvernement genevois, la Société suisse pour la protection de l'environnement (SPE) a établi un projet d'agenda 21 pour Genève ${ }^{8}$ en définissant, en particulier, 21 actions prioritaires pour le canton. En Suisse alémanique, les cantons d'Argovie et de Bâle-Campagne examinent les possibilités d'élaboration d'un plan d'action cantonal.

\section{Les indicateurs du développement durable}

Le chapitre 40 de l'Agenda 21, «Information pour la prise de décision», vise à l'élaboration d'indicateurs de développement durable (IDD) et cherche à en promouvoir l'utilisation. Seul l'établissement d'indicateurs intégrant des informations environnementales, démographiques, sociales, économiques et de développement permettra d'établir si les activités humaines conduisent à promouvoir une société durable. Reste que le système d'indicateurs doit encore être précisé.

\footnotetext{
5. www.agenda21local.ch.

6. OFEFP, Agenda 21 locaux. Pour un développement durable en Suisse, OFEFP, Berne, 1999.

7. Comité de candidature Sion 2006, Le livre Arc-en-ciel du développement durable, Sion, automne 1998.

8. L'agenda 21 pour Genève comporte 12 cahiers thématiques consultables sur Internet (www.geneve.ch/agenda21), ainsi qu'un rapport de synthèse présentant les 21 actions prioritaires.
} 
De nombreuses contraintes pèsent sur sa définition; le système d'indicateurs doit être performant et coordonné sur le plan international, consensuel, et doit donner une image représentative des trois dimensions du développement durable (aspects sociaux, environnementaux et économiques). En outre, ces indicateurs doivent être comparables entre pays et établis sur une base scientifique et technique solide.

La Commission pour le développement durable (CDD) a établi une liste de 134 indicateurs afin d'obtenir une base d'information homogène dans un grand nombre de pays dont le développement est différent, ce qui doit également faciliter l'évaluation de la mise en œuvre de l'Agenda 21 à l'échelon global et pays par pays 9 .

En Suisse, l'Office fédéral de l'environnement, des forêts et du paysage (OFEFP) et 1'Office fédéral de la statistique (OFS) ont lancé un projet pilote visant à

๖ évaluer et présenter une première série limitée d'indicateurs de développement durable adaptés à la Suisse et représentatifs des trois dimensions sociale, économique et environnementale;

- susciter la discussion avec les milieux intéressés afin de déterminer leurs besoins et leurs attentes;

- recueillir les expériences nécessaires à la réalisation d'un système plus évolué d'indicateurs de développement durable.

Il n'existe pas encore d'instrument qui permette de mesurer dans tous les domaines les progrès réalisés par la Suisse sur la voie du développement durable. Pour combler cette lacune, les deux offices proposent un choix d'indicateurs, tous issus de la liste des 134 indicateurs de la CDD, qui devraient permettre de donner une vue d'ensemble nécessaire à un suivi systématique.

L'étude pilote, éditée par l'Office fédéral de la statistique ${ }^{10}$, explique les raisons du choix de ces 33 indicateurs, qui sont présentés dans le détail. Cette publication a pour dessein d'ouvrir le débat avec les milieux intéressés. Une décision définitive quant au panel d'indicateurs pour le développement durable sera prise après une vaste consultation durant l'hiver 1999-2000.

\section{Conseil du développement durable}

Le Conseil du développement durable, organe consultatif du Conseil fédéral, a été créé en 1998. Chargé d'évaluer la mise en œuvre du développement durable en Suisse, il est également un organe d'impulsion appelé à formuler des propositions pour orienter la société vers le développement durable. Durant les premiers mois de son activité, le conseil a émis deux prises de position: la première sur la politique énergétique de la Suisse, dans laquelle il encourage la fiscalité écologique et recommande de se fixer comme objectif une division par trois de la consommation d'énergie jusqu'en 2050; la deuxième sur les indicateurs de développement durable, dans laquelle il recommande de compléter la liste proposée par d'autres indicateurs économiques et sociaux et s'engage à veiller à leur mise en œuvre.

9. United Nations, Indicators of Sustainable Development: Framework and Methodologies, New York, 1996.

10. OFEFP/OFS, Le développement durable en Suisse. Eléments pour un système d'indicateurs. Une étude pilote selon la méthodologie de la Commission du développement durable des Nations Unies, OFS, Neuchâtel, 1999. 


\section{3 indicateurs de développement durable retenus pour la Suisse}

\section{Indicateurs sociaux}

Bien-être et pauvreté matériels, éducation, utilisation de l'espace, urbanisation

1. Indice Gini sur la répartition des revenus

2. Surface habitable par personne

3. PIB consacré à l'éducation

4. Durée de scolarité

5. Taux de migration nette

6. \% de la population dans les zones urbaines

7. Surface et population de zones urbaines

\section{Indicateurs économiques}

Performance économique, habitudes de consommation, énergie
8. PIB par habitant
9. Dette par rapport au PIB
10. Taux de chômage
11. Consommation d'énergie par habitant
12. Consommation d'énergie renouvelable
13. Consommation de carburants pour les transports
14. Aide publique au développement

\section{Indicateurs environnementaux}

Eau, air, sol, agriculture, diversité biologique, déchets et déchets radioactifs

15. Consommation d'eau par habitant

16. Demande biochimique en oxygène

17. Emissions de gaz à effet de serre

18. Emissions d'oxydes d'azote

19. Substances appauvrissant l'ozone

20. Pollution des zones urbaines

21. Changements d'utilisation des sols

22. Terres arables par habitant

23. Terrains contaminés par les déchets spéciaux

24. Utilisation de pesticides

25. Utilisation d'engrais

26. Utilisation d'énergie pour l'agriculture

27. Superficie à protéger/superficie totale

28. Espèces menacées/espèces indigènes

29. Elimination des déchets ménagers

30. Recyclage et réutilisation des déchets

31. Importation et exportation de déchets spéciaux

32. Production de déchets spéciaux

33. Production de déchets radioactifs

Source: OFEFP/OFS, Le développement durable en Suisse. Eléments pour un système d'indicateurs, OFS, Neuchâtel, 1999.

\section{SOURCES}

Newsletter de la DDC, «CDD-7, la Suisse peut être satisfaite», juillet 1999.

Bulletin des négociation de la Terre, vol. 5, n' 1332, mai 1999.

E\&D File, «Commission on Sustainable Development, Seventh Session», vol. III, n 19, July 1999.

Environnement, «Du concept global à l'Agenda 21 local», bulletin d'information de l'OFEFP 4/98.

SITES INTERNET

Secrétariat de la Commission du développement durable (CDD) : www.un.org/esa/sustdev/csd.htm. Bulletin des négociations de la Terre: www.iisd.ca/french/index.html.

Site de l'OFEFP consacré aux agendas 21 locaux en Suisse: www.agenda21local.ch.

Agenda 21 pour le canton de Genève: www.geneve.ch/agenda21. 


\subsection{MISE EN EUVRE DE LA CONVENTION SUR LES CHANGEMENTS CLIMATIQUES}

La Convention sur les changements climatiques signée à Rio en 1992 est une convention-cadre contenant plus de déclarations de principe que d'engagements précis. Ces derniers se négocient, pas à pas, lors des conférences des parties (COP) qui se tiennent environ chaque année depuis l'entrée en vigueur de la convention (mars 1994) ${ }^{\prime \prime}$. Ce chapitre présente les travaux des $4^{e}$ et $5^{e}$ Conférences des parties (COP-4 et COP-5) à la Convention sur les changements climatiques qui ont eu lieu respectivement en automne 1998 et 1999 afin, notamment, de mettre en auvre le Protocole de Kyoto. Le bilan de la COP-4, qui s'est tenue à Buenos Aires en novembre 1998, est très mitigé. Le résultat de la conférence est un plan d'action définissant les grandes étapes de la mise en cuvre de la convention et du protocole jusqu'à la fin de l'an 2000. La $5^{e}$ Conférence des parties, qui s'est tenue à Bonn en novembre 1999, s'est déroulée dans une meilleure atmosphère politique: bien qu'aucune décision majeure n'ait été prise, l'agenda des négociations politiques a pu être précisé. Sur le plan suisse, la Confédération participe à la phase pilote des activités de mise en æuvve conjointe. Parallèlement, la politique énergétique de la Suisse est à un tournant. La nouvelle loi sur l'énergie est entrée en vigueur début 1999. Une nouvelle loi, dite sur le $\mathrm{CO}_{2}$, a été approuvée par le Parlement en octobre 1999 et la loi sur le marché de l'électricité est en discussion. Enfin, trois initiatives populaires ont poussé le Parlement et le gouvernement helvétiques à proposer une réforme fiscale écologique.

\section{$\square$ Le Protocole de Kyoto}

La $1^{\text {re }}$ Conférence des parties, qui s'est tenue à Berlin en avril 1995, a permis d'ouvrir les négociations d'un protocole visant la réduction des gaz à effet de serre. Le protocole, qui contient des objectifs de réduction pour les pays industrialisés seulement, a finalement été adopté deux ans et demi plus tard au terme de négociations difficiles, lors de la $3^{\text {e }}$ Conférence des parties à Kyoto (COP-3) en décembre $1997^{12}$.

Lors de la COP-3, les pays industrialisés se sont engagés de manière contraignante sur une base chiffrée. Aucun pays industrialisé n'a, pour l'instant, ratifié le protocole, qui n'est donc pas encore en vigueur. Ils contrôleront les émissions de six gaz à effet de serre pour parvenir, sur les années 2008 à 2012, à une réduction moyenne de leurs émissions d'au moins 5,2\% par rapport aux émissions de 1990. Tous les Etats n'ont pas les mêmes objectifs: la Suisse et l'Union européenne prise globalement se sont engagées à diminuer leurs émissions de $8 \%$ chacune, les Etats-Unis de 7\%, le Japon de 6\%, alors que la Russie et l'Ukraine doivent les stabiliser au niveau de 1990 et que l'Australie peut les augmenter de $8 \%$ !

Le Protocole de Kyoto prévoit en outre des mécanismes dits de souplesse ou de flexibilité donnant aux pays industrialisés la possibilité d'atteindre une partie de ces réductions hors de leur territoire national. Ce sont ${ }^{13}$ :

11. Elle est ratifiée par 180 pays (situation en septembre 1999).

12. Le texte du Protocole de Kyoto et de la Convention sur les changements climatiques peut être consulté sur le site www.admin.ch/buwal/klima/f/klimapolitik international.htm.

13. Pour les deux premiers mécanismes (échange de droits d'émission et mise en œuvre conjointe), seuls les pays industrialisés (dits de l'Annexe I à la convention) sont autorisés à y participer. 
- l'échange de droits d'émission (international emission trading). Un régime international d'échange sera créé. Les pays ayant réduit leurs émissions audelà de l'objectif qui leur a été assigné pourront céder leurs excédents de droits à d'autres (art. 17);

- la «mise en œuvre conjointe» (joint implementation) des projets de protection du climat par des investisseurs et des partenaires de pays industrialisés dans les pays d'Europe de l'Est (art. 6);

- le «mécanisme pour un développement propre» (Clean development mechanism). Il s'agit du financement par des pays industrialisés de projets de réduction des émissions dans les pays en développement (art. 12).

Un des principes directeurs de la convention prévoit que «les politiques et mesures qu'appellent les changements climatiques requièrent un bon rapport coût-efficacité, de manière à garantir des avantages globaux au coût le plus bas possible» (art. 3 de la convention). Pour le système climatique, toute réduction des émissions de gaz est bénéfique, où qu'elle intervienne dans le monde. Les coûts marginaux des mesures de protection du climat étant vraisemblablement plus élevés dans les pays industrialisés (voir ci-dessous), il est plus avantageux de prendre des mesures à meilleur prix : ces instruments permettent précisément aux pays occidentaux de remplir leurs engagements en finançant des projets de protection du climat, à l'étranger, notamment dans les pays en développement (mécanisme pour un développement propre) et dans les pays de l'Est (mise en œuvre conjointe). Ces mécanismes leur permettront d'inscrire à leur compte des réductions d'émissions de gaz à effet de serre (voir ci-dessous la section consacrée au programme pilote suisse d'activités de mise en œuvre conjointe). Mais, comme le soulignent certaines $\mathrm{ONG}^{14}$, ces instruments ne sont pas exempts de critiques. En effet, si les pays industrialisés misent entièrement sur eux, le commerce des «droits de polluer» devient un moyen de se débarrasser du problème. Une autre critique concerne le «droit de propriété sur l'atmosphère» qu'aurait implicitement attribué le protocole aux pays industrialisés à travers ces mécanismes.

Ce protocole ne soumet pas les pays en développement à des objectifs ni à des échéances spécifiques. Il sera légalement contraignant lorsque 55 Etats, dont des pays industrialisés représentant au moins $55 \%$ des émissions, l'auront ratifié. En réalité, la condition des $55 \%$ permet à un petit nombre de pays agissant de concert, comme les Etats-Unis ou la Russie, de bloquer l'entrée en vigueur du protocole car la réalisation de ce critère des $55 \%$ est impossible sans leur participation.

Le Protocole de Kyoto a été ouvert aux signatures le 12 décembre 1997. Plus de 80 Etats l'ont signé, dont la Communauté européenne et la Suisse, mais aucun pays industrialisé ne l'a encore ratifié. En novembre 1999, seuls 16 Etats, tous en voie de développement, l'avaient ratifié. Pour la Suisse, le principe de la ratification est acquis. Cependant, elle attendra que la Conférence des parties adopte les décisions et les modes d'application. Cette ratification ne nécessitera aucune modification de la législation suisse puisque la loi $\mathrm{CO}_{2}$ a été adoptée par le Parlement en automne 1999.

14. Communauté de travail, «Energie, la Suisse à un tournant», Revue Sud, 6/1998. 
Ces négociations ont été marquées par le traditionnel antagonisme Nord-Sud. Les pays en développement ont souligné la responsabilité des pays industrialisés dans le renforcement de l'effet de serre et ont estimé que c'est à eux de supporter le coût de la lutte contre le réchauffement planétaire. Les pays industrialisés ont, pour leur part, signifié leur inquiétude face à la croissance rapide des émissions dans les pays du Sud et leur demande à propos d'un engagement formel pour contrôler cette progression. Cette revendication s'est traduite par la décision du Congrès et du Sénat américains, votée en 1997, de ne pas ratifier le protocole sans un accord sur la participation des pays en développement. Mais une autre opposition traverse les débats, qui concerne les Etats-Unis d'une part, et la Communauté européenne et la Suisse d'autre part. Les Américains rechignent à limiter les émissions par des réglementations domestiques, mais misent plutôt sur les «mécanismes de flexibilité». Les Européens entendent au contraire placer la priorité sur des mesures de limitations domestiques (par opposition aux mécanismes qui permettent d'obtenir ces déductions à l'étranger), comme la fiscalité écologique, et ne recourir aux mécanismes de Kyoto que dans une mesure limitée.

\section{De la signature à la mise en œuvre d'une convention internationale}

Les conventions-cadres sur les changements climatiques et la diversité biologique, qui ont été signées lors de la Conférence des Nations Unies pour l'environnement et le développement en juin 1992 à Rio, ont marqué le début d'un processus qui se poursuit depuis lors. D'année en année, I'Annuaire SuisseTiers Monde suit et résume les négociations qui ont lieu lors des conférences des parties afin de préciser la mise en œuvre des deux traités.

Signature et ratification d'un traité international. La signature d'un traité est un acte solennel qui est le point terminal de la procédure de négociation au cours de laquelle les Etats sont intervenus. La signature démontre la préoccupation d'un Etat envers une thématique, mais ne l'engage pas juridiquement. Le traité ne devient obligatoire pour les Etats que s'il est ratifié. En Suisse, le Conseil fédéral a l'autorité pour signer un traité, mais c'est le Parlement qui le ratifie.

Entrée en vigueur d'un traité international. Un traité international entre en vigueur après le dépôt des instruments de ratification d'un certain nombre d'Etats. Généralement, le nombre de ratifications nécessaires ainsi que les modalités propres à chaque convention sont précisés dans le texte du traité en question.

Les conférences des parties. Une fois le traité international entré en vigueur, les Etats parties au document, c'est-à-dire ceux qui l'ont ratifié, se rencontrent à intervalles réguliers pour mettre le texte en œuvre. Dans de nombreux cas, les textes sont des conventions-cadres qui nécessitent des négociations complémentaires afin d'en préciser la mise en œuvre. Ces réunions sont connues sous l'expression "conférence des parties à la convention $X$ ou $Y$ " et sont numérotées. Dans le jargon diplomatique et juridique, on utilise des abréviations devenues maintenant courantes. Ainsi, la $4^{\mathrm{e}}$ Conférence des parties à la Convention-cadre sur les changements climatiques, qui s'est tenue à Buenos Aires en novembre 1998, est également connue sous le sigle COP-4 CCCC.

\section{$\square$ La 4 Conférence des parties (COP-4), Buenos Aires (2-13 novembre 1998)}

La $4^{\mathrm{e}}$ Conférence des parties (COP-4) à la Convention sur les changements climatiques s'est tenue du 2 au 13 novembre 1998 à Buenos Aires, en présence de plus de 5000 participants. La délégation suisse, conduite par Philippe Roch, directeur de l'OFEFP, était composée de collaborateurs des Offices fédéraux de 
l'environnement, des forêts et du paysage, des affaires étrangères et des affaires économiques extérieures. Philippe Roch a représenté la Suisse lors du segment ministériel.

Lors du segment du haut niveau, les ministres présentèrent des aperçus sur les actions nationales entreprises dans le domaine des changements climatiques. Au nom de la Suisse, Philippe Roch a évoqué l'approbation, par l'une des Chambres du Parlement suisse, d'une loi prévoyant la réduction de $10 \%$ des émissions de $\mathrm{CO}_{2}$ en 2010 par rapport au niveau de $1990^{15}$. Il a rappelé la proposition faite par la Suisse lors de la présente conférence d'envisager conjointement les gaz visés par les Protocoles de Kyoto et de Montréal. En effet, certains gaz présentés comme alternatifs aux CFC dans le cadre du Protocole de Montréal ont un potentiel de réchauffement global élevé. Le directeur de l'OFEFP a enfin souligné la responsabilité des pays industrialisés et estimé que le «mécanisme pour un développement propre» était un instrument très prometteur.

\section{Les résultats de la conférence de Buenos Aires}

La conférence de Buenos Aires s'est achevée par l'adoption d'un plan d'action dans lequel les parties réitèrent leur volonté de mettre en œuvre la convention et de préparer la future entrée en vigueur du Protocole de Kyoto. Le document contient également des décisions dans divers domaines: mécanismes de financement, transferts de technologie, questions concernant les répercussions économiques négatives des changements climatiques et des mesures de protection du climat pour les pays en développement, phase pilote des projets conjoints, mécanismes du Protocole de Kyoto, ainsi que la préparation de la première conférence des parties une fois le protocole entré en vigueur. Le programme d'action fixe également les étapes nécessaires à la concrétisation des trois instruments (mécanismes de souplesse) jusqu'à la fin de l'an 2000.

Deux événements importants ont marqué le déroulement de la conférence. Le premier revient à l'Argentine, pays hôte de la conférence, qui a décidé de quitter les rangs des pays en développement pour signaler sa volonté de prendre, lors de la prochaine conférence des parties (COP-5), un engagement contraignant à réduire ses émissions de gaz à effet de serre. Par cette déclaration, l'Argentine a brisé un tabou, celui des engagements volontaires pour les pays en développement à réduire leurs émissions. Le second événement est survenu moins de vingt-quatre heures plus tard, avec la signature du Protocole de Kyoto par les Etats-Unis. Lors d'une conférence de presse, le secrétaire d'Etat américain Eizenstat a qualifié la décision du président Menem d'historique et a signalé que l'initiative argentine représentait le type de participation significative par les pays en développement qui préconditionne la ratification du protocole par les Etats-Unis.

\section{Evaluation de la conférence de Buenos Aires}

Les résultats de la Conférence de Buenos Aires divisent les différents acteurs. Pour les uns, les discussions ont abouti à un progrès minime, voire inexistant; pour les autres, des résultats significatifs ont malgré tout été obtenus. Philippe Roch se range dans la deuxième catégorie; à la clôture de la conférence, il affirmait: «Au sortir de la conférence de Kyoto, le plus grand flou régnait quant aux mesures à mettre en œuvre pour parvenir à remplir les objectifs clairs de 
réduction des gaz à effet de serre. A Buenos Aires, nous nous sommes tous mis d'accord sur un programme de travail clair qui doit aboutir, pour fin $2000 \mathrm{ou}$ début 2001, à l'instauration d'outils précis permettant cette réduction. C'est indéniablement un progrès. ${ }^{16}$ Les organisations non gouvernementales, quant à elles, n'ont pas ménagé leurs critiques. Les Amis de la Terre ont qualifié le résultat de «plan d'inaction» et la réunion de Buenos Aires de «foire de marchands cherchant à sauver l'industrie des combustibles fossiles». Greenpeace a déploré que la conférence «se soit transformée en négociation commerciale et économique au détriment de la science ${ }^{17}$.

\section{$\square$ La $5^{e}$ Conférence des parties (COP-5), Bonn (25 octobre-5 novembre 1999)}

La $5^{\text {e }}$ Conférence des parties s'est tenue à Bonn du 25 octobre au 5 novembre 1999. Lors de cette rencontre, à laquelle ont participé plus de 4000 délégués de 163 Etats, les négociations ont surtout porté sur les aspects techniques et politiques des mécanismes prévus par le Protocole de Kyoto. La délégation suisse, conduite par Philippe Roch, directeur de l'OFEFP, était composée de représentants de l'Office de l'environnement, du Seco, de l'Office fédéral de l'énergie et du Département des affaires étrangères, ainsi que de trois représentants non gouvernementaux des milieux scientifiques, économiques et des organisations d'environnement et d'entraide (Ecole polytechnique de Zurich, Vorort et WWF Suisse).

Selon le mandat défini par le Conseil fédéral, la délégation suisse s'est attachée à assurer une utilisation crédible des instruments économiques. Elle a défendu la nécessité de maintenir un équilibre raisonnable entre les réductions qui peuvent être effectuées par les Etats hors de leurs frontières et celles qu'ils doivent réaliser sur le territoire national. La Suisse s'est engagée pour qu'un régime fort de vérification du respect des dispositions soit mis en place. La délégation suisse a également présenté la nouvelle loi sur le $\mathrm{CO}_{2}$ adoptée par le Parlement en octobre 1999 (voir ci-dessous). Celle-ci prévoit une réduction des émissions nationales de $\mathrm{CO}_{2}$ de $10 \%$ par rapport à 1990 , dès 2010. La Suisse est le premier pays à prendre des mesures nationales se référant expressément au Protocole de Kyoto ${ }^{18}$.

Plus d'une centaine de ministres ont participé au segment ministériel de la conférence. Dans sa déclaration officielle, le chancelier allemand Gerhard Schroeder a engagé les gouvernements à ratifier rapidement le Protocole de Kyoto de façon à ce qu'il puisse entrer en vigueur en 2002, soit dix ans après le Sommet de Rio. Cette date butoir a insufflé l'élan politique nécessaire qui devrait permettre une ratification rapide du Protocole de Kyoto. Les délégations ont également adopté le calendrier des futures négociations, qui devront aboutir lors de la prochaine conférence des parties, qui se tiendra à La Haye en novembre 2000 (COP-6). Le secrétaire exécutif de la Convention sur le climat, Michael Zammit Cutajar, a qualifié le climat politique de bien meilleur que ce qui était attendu. Il a également relevé l'engagement et la volonté des ministres présents d'aboutir à des résultats concrets ${ }^{19}$. La délégation suisse s'est elle aussi déclarée «très satisfaite» des résultats de la COP-5, soulignant que la compré-

16. «La Suisse optimiste après le Sommet sur le climat», Le Temps, 17.11.98.

17. "La planète se réchauffe, Buenos Aires n’y changera rien », Le Temps, 16.11.98.

18. DETEC, «COP-5, Bonn. Réduire les gaz à effet de serre entre pays industrialisés et pays en développement», «La délégation suisse est satisfaite», Communiqués de presse, 4.10.99 et 5.11.99.

19. UNFCC, «Ministers Pledge to Finalize Climate Agreement by November $2000 »$, Press Release, 5.11.99. 
hension entre les partenaires et l'esprit de coopération ont beaucoup progressé depuis Kyoto et Buenos Aires.

\section{$\square$ Le programme pilote suisse d'activités de mise en œuvre conjointe}

Dès 1996, la Suisse a fait part de son intention de participer à la phase pilote des activités de mise en œuvre conjointe (AIJ). Le programme suisse a été lancé pour une période de trois ans en février 1997 sous l'abréviation SWAPP (Swiss AIJ Pilot Program $)^{20}$. Le secrétariat du SWAPP est intégré au Secrétariat de l'économie (Seco) et un comité interdépartemental, composé de représentants des Offices de l'environnement et de l'énergie, de la Direction du développement et de la coopération et du Seco, en supervise les activités ${ }^{21}$.

Le programme pilote suisse a les objectifs suivants:

- acquérir de l'expérience dans l'utilisation des AIJ;

- contribuer à réduire les émissions de gaz à effet de serre et à mettre en œuvre la Convention sur les changements climatiques;

- tirer parti du potentiel efficacité-coût des instruments de mise en œuvre conjointe avec les pays en développement (mécanisme pour un développement propre) et avec les pays de l'Est (mise en œuvre conjointe) dans le cadre de la politique suisse sur le climat;

๑ réaliser des projets modèles;

- promouvoir les transferts de technologie pour limiter les émissions de gaz à effet de serre et encourager les investissements du secteur privé;

- contribuer au développement de méthodes crédibles permettant de quantifier et vérifier les réductions d'émissions effectives.

Les activités conduites par le SWAPP

Les activités du SWAPP sont menées sur divers fronts pour tirer le meilleur parti de la phase pilote ${ }^{22}$. Depuis l'été 1999, un projet est en cours en Roumanie, qui prévoit l'assainissement de deux réseaux de chauffage à distance dans deux villes roumaines, ainsi que l'installation de nouvelles centrales thermiques. Pendant les quinze ans de durée du projet, on estime pouvoir éviter l'émission de 140 '000 tonnes de $\mathrm{C}_{2}$. Financé à hauteur de 5,5 millions de francs par la Confédération, ce projet est également financé par la Roumanie, qui prend en charge les frais locaux. Parmi d'autres activités du SWAPP, la Suisse soutient, conjointement avec la Banque mondiale, un projet ayant pour but d'aider les gouvernements des pays qui pourraient bénéficier des activités de mise en œuvre conjointe à saisir les chances qui s'offrent à eux avec ces nouveaux instruments.

Durant cette phase pilote, le gouvernement suisse cherche également à encourager les investissements du secteur privé dans des projets de réduction des émissions $^{23}$. Deux tables rondes Confédération-secteur privé ont été organisées (en janvier et novembre 1999) afin de présenter aux entreprises suisses les mécanismes de souplesse et de les inviter à s'engager dans les activités de mise en œuvre conjointe (AIJ). A ce jour, il est difficile d'estimer l'intérêt des entre-

20. Le site Internet très complet du SWAPP (www.admin.ch/swissaij) présente le concept des AIJ ainsi que les activités du programme.

21. SWAPP, Dossier de presse AIJ Pilot Program, janvier 1999. Sur Internet: www.admin.ch/swissaij/pdf/in-pdswapp99f.pdt.

22. Une vue d'ensemble des différentes activités du SWAPP est disponible sur Internet: www.admin.ch/swissaij/activitiesbody.html.

23. Le compte-rendu d'une table ronde entre la Confédération et le secteur privé sur les AIJ peut être consulté à l'adresse Internet suivante: www.admin.ch/swissaij/in_rt_summary.html. 
prises helvétiques pour ces nouveaux mécanismes, qui dépendra de l'obligation qu'elles auront de réduire leurs émissions après l'adoption de la loi sur le $\mathrm{CO}_{2}$, ainsi que du cadre international qui se précisera lors de la $6^{\mathrm{e}}$ Conférence des parties en 2001. Les mécanismes de souplesse du Protocole de Kyoto présupposent que les coûts marginaux des mesures de protection du climat sont plus élevés dans les pays industrialisés. Mais au stade actuel de la discussion, il s'agit encore une hypothèse puisque les coûts de leur mise en œuvre n'ont pas encore été évalués (inclusion du prix dans la transaction, arrangements institutionnels, etc.). Les entreprises suisses sont donc attentives à l'évolution du débat, espérant pouvoir bénéficier par les mécanismes de souplesse d'un moindre coût dans la réduction des émissions à l'étranger. Un postulat, déposé en juin 1998 par G.-R. Plattner, conseiller d'Etat socialiste bâlois, demande au Conseil fédéral une évaluation des instruments du Protocole de Kyoto. Le Conseil fédéral a accepté ce postulat et un rapport sera présenté au Parlement à la fin de l'an 2000 sur les possibilités de mise en application et les potentialités des nouveaux instruments d'économie de marché pour la protection du climat. Le rapport tentera également de cerner l'intérêt des entreprises suisses pour ces dispositifs.

Lors du voyage au Brésil du conseiller fédéral Pascal Couchepin en juillet 1999, deux accords dans le domaine de la protection de l'environnement ont été signés. Un montant de 8,6 à 11,8 millions de francs est budgété à cet effet pour les cinq ans à venir. Le premier accord consiste en une étude conjointe entre les deux pays pour tenter d'analyser les questions que pose encore le concept de «mécanisme pour un développement propre» (ce soutien s'inscrit dans le cadre du projet Suisse/Banque mondiale $)^{24}$. Le second accord porte sur la création commune d'un «Cleaner Production Center», basé à São Paulo. Ce centre est destiné à améliorer les techniques de production dans l'industrie et à accroître l'efficacité écologique des méthodes de transformation plus respectueuses de l'environnement ${ }^{25}$. A noter que trois autres centres du même type financés par la Suisse sont déjà en activité en Colombie, au Salvador et au Costa Rica.

\section{La politique climatique de la Suisse}

Avec des émissions annuelles d'un peu plus de 6 tonnes de $\mathrm{CO}_{2}$ par tête d'habitant, la Suisse dépasse largement l'objectif de 1 à 2 tonnes considéré par les milieux scientifiques comme globalement supportable à long terme du point de vue climatique. Elle est bien placée par rapport aux pays de l'OCDE, puisque la moyenne des pays industrialisés se situe autour de 11 tonnes, alors que la moyenne mondiale est de 4 tonnes par habitant.

Pour améliorer cette situation et respecter les engagements pris au titre de la Convention sur le climat et du Protocole de Kyoto, la Suisse met l'accent sur les mesures internes visant à limiter l'utilisation des combustibles et des carburants fossiles. Ainsi, la politique climatique de la Suisse repose sur les politiques énergétique, environnementale, des transports, agricole et forestière, ainsi que sur une réforme fiscale écologique.

La nouvelle loi sur l'énergie est entrée en vigueur le $1^{\text {er }}$ janvier $1999^{26}$. Cette loi vise un approvisionnement en énergie économique et compatible avec les impé-

24. Seco, «Protection du climat. La Suisse et le Brésil s'associent», Communiqué de presse, 7.7.99.

25. Seco, «La Suisse lance un Cleaner Production Center au Brésil», Communiqué de presse, 7.7.99.

26. Message du 21 août 1996 concernant la loi sur l'énergie (LEn). 
ratifs écologiques, l'utilisation économe et rationnelle de l'énergie, ainsi qu'une utilisation accrue des énergies indigènes et renouvelables.

En automne 1999, le Parlement a adopté la loi sur le $\mathrm{CO}_{2}$, élément essentiel de la politique climatique suisse. La loi sur le $\mathrm{CO}_{2}$ est un parfait exemple d'intégration des objectifs économiques et des préoccupations environnementales. Elle tente de responsabiliser les acteurs concernés et de favoriser une meilleure coopération entre l'Etat et les milieux économiques, et mise sur les incitations plutôt que sur des obligations ou interdictions.

Les principaux éléments de la loi sur le $\mathrm{CO}_{2}$ sont les suivants:

- fixation d'un objectif de réduction des émissions de $\mathrm{CO}_{2}$ de $10 \%$ d'ici à 2010 par rapport à leur niveau de 1990;

- prise en considération des mesures déjà adoptées ou prévues par la Confédération, qui contribuent à réduire les émissions de $\mathrm{CO}_{2}$;

a prise en considération des mesures librement consenties par les milieux économiques;

๖ introduction au plus tôt en 2004 d'une taxe sur le $\mathrm{CO}_{2}$ s'il est prévisible que les autres mesures ne suffiront pas à atteindre les objectifs fixés;

$\checkmark$ exonération des entreprises s'engageant à réaliser des réductions volontaires de $\mathrm{CO}_{2}$;

๑ possibilité d'utilisation des instruments internationaux conformément au Protocole de Kyoto.

Par l'adoption de cette loi, la Suisse dispose des bases légales nécessaires à la mise en œuvre des engagements pris à Kyoto. Elle est le premier pays qui stipule dans sa législation que les objectifs de Kyoto doivent être atteints.

Mais nombreux sont les projets en chantier qui affecteront la politique climatique. En octobre 1998, le Conseil fédéral a élaboré un panorama général de sa future politique énergétique. Il s'est prononcé pour une réforme fiscale écologique à moyen terme et pour une taxe sur l'énergie à court terme ${ }^{27}$. La loi sur le marché de l'électricité (LME) est en cours d'élaboration. De plus, en juin 1999 le Conseil fédéral a annoncé une refonte de la législation sur l'énergie atomique. Trois initiatives sont pendantes: l'initiative «solaire», l'initiative «énergie et environnement» et l'initiative «Taxer l'énergie et pas le travail». Durant la session d'automne 1999, le Parlement a décidé de deux contre-projets à opposer aux initiatives populaires. Pour ce qui est du contre-projet direct à l'initiative «énergie et environnement» (introduction d'une norme fondamentale dans la Constitution en vue de taxer les énergies non renouvelables, estimée à plus de 3 milliards de francs par année), le Parlement propose que le produit de la taxe, dite taxe d'incitation, qui s'élèverait à 2 centimes par kilowattheure, vise exclusivement à compenser les charges salariales annexes payées par les employeurs et les salariés. Ce contre-projet, qui constitue la première étape de la réforme fiscale écologique, a été accepté par le Parlement. Il en va de même du contreprojet à l'initiative «solaire», conçu sous la forme d'une disposition transitoire,

27. DETEC/DFF, «Une taxe sur l'énergie devrait précéder l'intégration d'incitations écologiques dans le nouveau régime financier", Communiqué de presse, 28.10.98. 
une taxe dite d'encouragement qui mettra en place un régime de subvention de 450 millions de francs par année ${ }^{28}$.

Le peuple suisse sera vraisemblablement appelé à se prononcer sur les initiatives et leurs contre-projets au cours de l'an 2000.

[C] ASTM 1998, «Mise en œuvre de la Convention sur les changements climatiques», pp. 209-213.

\section{La réforme fiscale écologique}

La réforme fiscale écologique est au cœur des débats politiques en Suisse, comme l'atteste une série de projets: trois initiatives populaires, des propositions du Conseil national et du Conseil des Etats, et les travaux du Conseil fédéral. L'objectif principal est de taxer davantage les énergies non renouvelables: pétrole, gaz, charbon, uranium. Le prix très abordable de l'énergie n'encourage pas une consommation économe. En prix réel, les combustibles sont moins chers que jamais. L'objectif premier des autorités fédérales est de privilégier le concept de la réforme fiscale purement écologique. Les revenus de la taxe énergétique ne doivent pas augmenter les revenus de l'Etat, mais permettre de réduire les charges salariales; les entreprises paieront certes l'énergie plus cher, mais le travail sera moins taxé. A long terme, l'économie devrait même profiter d'une meilleure efficacité énergétique.

La Société suisse pour la protection de l'environnement (SPE) a publié un fascicule de référence faisant le point sur les fondements et les instruments d'une réforme fiscale écologique.

Sources: Environnement, "Taxer l'énergie plutôt que le travail», bulletin d'information de I'OFEFP, 1/99.

SPE, En marche vers une réforme fiscale écologique, SPE, Genève, octobre 1999.

\section{SOURCES}

OFEFP, Communiqués de presse publiés en 1999.

OFEFP, Fiches d'information publiées à l'occasion de la 4 Conférence des parties - Buenos Aires (octobre 1998) et de la $5^{e}$ Conférence des parties - Bonn (octobre 1999) à la Convention sur les changements climatiques.

PNUE, «Buenos Aires 1998», Dossier de presse, PNUE, novembre 1998.

ONU, Secrétariat de la Convention-cadre sur les changements climatiques, communiqués de presse parus en 1998 et 1999.

E\&D File, «UN Framework Convention on Climate Change: COP-4», United Nations non Governmental Liaison Service/NGLS, vol. III, no. 18, Dec. '98-Jan. '99.

Environnement, «Les petits ruisseaux font les grandes rivières. Bilan suisse de la conférence de Buenos Aires», bulletin d'information de l'OFEFP, 1/99.

Courrier de la Planète, dossier «Le climat risque majeur et enjeu politique. De la conférence de Kyoto à celle de Buenos Aires", Solagral et Global Change, mars-avril 1998.

Bulletin des négociations de la Terre, vol. 12, $\mathrm{n}^{\circ}$ 97, juin 1999.

Swiss AIJ Pilot Program, «Les mécanismes de flexibilité du Protocole de Kyoto et le programme Suisse AIJ», Dossier de presse, janvier 1999.

SDES, Dossiers thématiques $n^{\circ} 11$, Politique énergétique, édition 1999.

Communauté de travail, «Energie, la Suisse à un tournant. Plaidoyer pour une réforme fiscale écologique», Revue Sud, $6 / 1998$.

\section{SITES INTERNET}

Convention sur les changements climatiques: www.unfccc.de.

Site du PNUE consacré aux conventions internationales de protection de l'environnement: www.unep.ch/iuc.html OFEFP: /www.admin.ch/buwal/.

Bulletin des négociations de la Terre: www.iisd.ca/french/index.html.

Swiss AIJ Pilot Program: www.admin.ch/swissaij.

SDES (pipeline politique - dossiers thématiques): www.politique.ch/index.htm.

28. Il est important de distinguer la taxe d'encouragement en matière énergétique de 0.3 centime par kilowattheure, avec une durée maximale de quinze ans (taxe transitoire sur les énergies non renouvelables), de la taxe d'incitation (conçue sous forme d'une norme constitutionnelle), qui s'élèvera à 2 centimes par kilowattheure au maximum. 


\subsection{MISE EN EUUVRE DE LA CONVENTION SUR LA BIODIVERSITÉ}

Les gouvernements de plus de 130 pays se sont réunis à Cartagena en février 1999 dans le but de signer un Protocole sur la biosécurité. Cependant, après dix jours de négociations, les délégués n’ont pas réussi à finaliser le document et la conférence s'est terminée sur un échec. La Suisse, présente lors des négociations, a créé et présidé un groupe de compromis pour tenter de sortir de l'impasse, mais sans succès.

\section{$\square$ Conférence extraordinaire des parties (février 1999)}

La première réunion extraordinaire de la Conférence des parties (COP-ex) de la Convention sur la diversité biologique s'est déroulée les 22 et 23 février 1999 à Cartagena (Colombie); plus de 600 participants représentant 138 gouvernements, des ONG, le secteur industriel et la communauté scientifique y ont pris part. Les Etats parties à la convention devaient adopter un protocole international sur la sécurité dans le domaine de la biotechnologie (biosécurité). Dix jours d'intenses négociations avaient précédé la COP-ex, les délégués devant fournir un texte finalisé prêt pour une adoption formelle ${ }^{29}$. Le texte n'a pas obtenu de consensus et la conférence s'est terminée sur un échec.

La délégation suisse, conduite par l'ambassadeur Beat Nobs (OFEFP), comprenait des représentants des Offices fédéraux de l'environnement, des affaires étrangères et des affaires économiques extérieures.

\section{$\square$ Protocole sur la biosécurité}

La décision d'instituer un Protocole sur la biosécurité a été prise lors de la COP-2 à Jakarta (novembre 1995). Ce protocole doit prévenir les risques biologiques potentiels liés aux mouvements transfrontières d'organismes vivants modifiés (OVM) ${ }^{30}$ susceptibles d'avoir des conséquences négatives sur la diversité biologique et l'environnement. Les discussions sur la prévention des risques biotechnologiques sont le reflet de l'inquiétude grandissante du public face aux risques des nouvelles biotechnologies, tel le génie génétique.

Les principales dispositions du Protocole sur la biosécurité en cours de négociation sont les suivantes:

- clauses préliminaires (préambule, objectifs, usage des termes);

๑ champs d'application du protocole;

- mécanisme d'accord préalable en connaissance de cause (AIA) ${ }^{31}$;

- évaluation et gestion des risques;

๑ mouvements transfrontières involontaires et mesures d'urgence;

- manipulation, transport, emballage et étiquetage;

29. Le GTSB est un «groupe de travail spécial sur la biosécurité» mandaté par la Conférence des parties. Il s'est réuni à Cartagena du 14 au 22 février pour la sixième fois (GTSB-6), dans le but de finaliser le protocole qui devait être soumis à la COP-ex.

30. L'expression «organismes vivants modifiés» (OVM) a été créée par la COP; elle fait référence aux plantes et animaux transgéniques, génétiquement modifiés, ainsi qu'aux microorganismes tels que les virus et les bactéries, souvent appelés organismes génétiquement modifiés (OGM).

31. Dans la plupart des textes faisant référence à la procédure d'accord préalable en connaissance de cause, l'abréviation anglaise AIA est utilisée (Advanced Informed Agreement). 
」 échange d'informations, renforcement des capacités, sensibilisation/participation du public;

- considérations socio-économiques;

๑ trafic illégal;

๑ responsabilité et indemnisation;

- mécanismes de financement et ressources financières;

- dispositions institutionnelles (secrétariat, organes subsidiaires, entrée en vigueur);

ป annexes:

- informations requises dans les notifications pour obtenir l'accord préalable en connaissance de cause;

- facteurs à prendre en compte pour l'évaluation des risques.

Tous les Etats ne sont pas encore dotés d'une législation nationale en la matière, même si la plupart des pays possédant des industries biotechnologiques disposent d'un cadre légal. En outre, il n'existe pas d'accord international juridiquement contraignant concernant le franchissement de frontières par des OVM, par le fait d'activités commerciales ou suite à une fuite ou à un rejet. L'objectif du protocole (article premier) est de contribuer à la garantie d'un niveau adéquat de protection dans le domaine du transfert, de la manipulation et de l'utilisation sans risque des OVM résultant de la biotechnologie moderne. Des références sont faites au principe de précaution et aux risques posés à la santé humaine. A ce stade de la négociation, l'article exclut les références aux impératifs socioéconomiques et aux produits dérivés pour se focaliser surtout sur les mouvements transfrontières. Aucun consensus n'a pu être atteint lors de la réunion de Cartagena; plusieurs pays en développement, par exemple, souhaitaient le maintien de références aux impératifs socio-économiques, à l'inverse de certains pays industrialisés.

Dès le début des négociations, d'importantes divergences sont apparues sur de nombreux points $\mathrm{s}^{32}$. Les premiers désaccords ont surgi déjà lors de la détermination du champ d'application du protocole ainsi qu'au sujet de la procédure d'accord préalable donné en connaissance de cause (AIA). La procédure AIA, élément essentiel du protocole, doit garantir aux pays importateurs un libre accès à toute l'information nécessaire permettant l'évaluation préalable des impacts que peuvent avoir les OVM sur l'environnement. Les pays ont convenu que les semences génétiquement modifiées destinées à être plantées et d'autres OVM qui seront disséminés dans l'environnement rentrent dans le champ du traité, mais ils sont en total désaccord pour déterminer quels autres organismes modifiés ou «produits de ceux-ci» doivent être soumis à la procédure.

Aucun consensus n'a pu être trouvé sur la délicate question de la distribution des compétences et des obligations entre le secteur privé et les gouvernements dans la mise en œuvre des aspects opérationnels du protocole. D'autres aspects, plus techniques, ont également fait buter les négociateurs: le niveau de détail des dispositions techniques en matière d'évaluation et de gestion des risques, la prise en compte des questions de responsabilité civile et d'indemnisation socio-

32. Tous les articles du protocole ainsi que les différents points de divergence entre les Etats parties à la négociation sont présentés sur le site du Bulletin des négociations de la Terre (www.iisd.ca/linkages/vol09/enb09117fhtml). 
économiques. Mais le principal point de divergence concerne les mesures à appliquer lors de l'exportation de produits agricoles contenant des OVM, destinés soit à la consommation, soit à une transformation (grains). Cet important aspect du commerce agricole international a des implications sur les dispositions relatives à l'information, y compris l'étiquetage, et sur les relations commerciales avec des Etats non-parties, ainsi que sur les relations avec d'autres accords internationaux, notamment les instruments de l'OMC.

\section{Positions des différents acteurs}

Le «groupe de Miami» ${ }^{33}$, alliance de six producteurs céréaliers, a mené un intense lobby contre la conclusion du protocole. Leur position intransigeante concernant la procédure AIA (ils veulent en exclure les organismes modifiés destinés à la consommation humaine et animale) constitue un des motifs de l'arrêt des négociations. L'Union européenne est, pour sa part, restée fermement attachée à l'identification des produits contenant des OVM.

La compatibilité des clauses du protocole avec d'autres obligations internationales, notamment les règles de l'OMC, a fait l'objet d'âpres négociations. Le groupe de Miami a défendu de manière très forte la compatibilité des dispositions du protocole avec l'Accord sur les mesures sanitaires et phytosanitaires $(\mathrm{SPS})^{34}$ négocié à l'OMC. D'autres Etats ont estimé que cette «clause de sauvegarde de l'OMC» donne aux règles commerciales internationales une trop grande emprise sur les objectifs environnementaux du protocole. L'Union européenne a introduit une proposition de compromis, qui visait à préciser dans le préambule que la mise en œuvre du protocole et les autres obligations internationales «s'appuient mutuellement» plutôt qu'une référence à la compatibilité. Aucun consensus n'a pu être trouvé.

Tout au long des négociations, la Suisse a, pour sa part, défendu une position médiane $^{35}$. D'une part, elle a exigé une transparence totale sur les produits importés (en Suisse, l'importation de tout produit contenant des OVM est soumise à autorisation auprès des offices fédéraux et la signalisation est obligatoire s'il s'agit d'organismes vivants comme les semences). D'autre part, la Suisse exige également un étiquetage spécifique pour les denrées alimentaires. Elle s'est par contre montrée beaucoup moins claire sur les questions de responsabilité dans le domaine du génie génétique, estimant que cette question doit être traitée ultérieurement. Malgré sa position plus en retrait sur ce dernier point, la Suisse souhaitait vivement que la conférence de Cartagena aboutisse à la signature d'un protocole et, pour tenter de sortir de l'impasse, elle a créé et présidé un «groupe de compromis» qui réunissait le Japon, le Mexique, la Corée, la Norvège et la Nouvelle-Zélande. Les réflexions issues de ce groupe devaient permettre de faire de nouvelles propositions, soutenues par l'ensemble des délégations à l'exception du «groupe de Miami».

Malgré l'effort diplomatique de la Suisse, les ONG d'environnement, à l'instar du WWF Suisse et de Greenpeace, n'ont pas manqué de critiquer la position

\footnotetext{
33. Le «groupe de Miami» est composé des Etats-Unis, du Canada - tous deux pèsent pour près de $90 \%$ sur le marché des céréales -, de l'Australie, de l'Argentine, du Chili et de l'Uruguay. A noter que les Etats-Unis n'ont pas ratifié la Convention sur la biodiversité. Officiellement, ils n'ont qu'un statut d'observateur et n'ont pas le droit de vote, ce qui ne les empêche pas d'être très efficaces dans leur travail de lobby.

34. Cet accord exige que les mesures commerciales liées à la santé ou à l'environnement ne soient pas plus restrictives pour le commerce qu'il n'est requis et qu'elles reposent sur des «principes scientifiques solides».

35. «Les écologistes attaquent la Suisse sur la biosécurité», Le Temps, 24.2.99.
} 
helvétique. Dans un communiqué de presse ${ }^{36}$, le WWF Suisse reproche notamment à la Suisse de s'aligner sur les gros exportateurs d'organismes génétiquement modifiés et de négliger les intérêts des pays du Sud.

\section{Ordonnances fédérales \\ pour l'amélioration de la sécurité dans l'utilisation des organismes génétiquement modifiés}

Le Conseil fédéral, soucieux d'assurer une plus grande sécurité dans l'utilisation des organismes génétiquement modifiés, a mis en vigueur en août 1999 trois ordonnances. Celles-ci introduisent une obligation de notifier, une procédure d'octroi d'autorisation ainsi que des mesures de prévention et de protection.

L'ordonnance sur I'utilisation confinée (OUC) et l'ordonnance sur la dissémination dans I'environnement (ODE) ont pour but de protéger l'homme et l'environnement. L'ordonnance sur la protection des travailleurs contre les risques liés aux microorganismes (OPTM) prévoit des mesures de sécurité particulières pour les personnes qui, sur leur lieu de travail, entrent en contact avec des microorganismes pathogènes ou génétiquement modifiés. Ces trois ordonnances, étroitement harmonisées entre elles, constituent les actes législatifs essentiels dans le domaine de la sécurité biologique.

Les points forts des nouvelles réglementations sont les suivants:

- évaluation du risque pour les activités dans les laboratoires de recherche et les installations de production;

- autorisation obligatoire pour les disséminations expérimentales et la mise en circulation d'OGM;

๑ interdiction de disséminations expérimentales;

- toute demande d'autorisation de dissémination doit être accompagnée d'un programme de surveillance de l'environnement (monitoring).

Les trois nouvelles ordonnances concrétisent les dispositions légales en vigueur dans le domaine de I'environnement et améliorent la protection de la santé sur le lieu de travail. D'autres prescriptions concernant l'utilisation d'OGM seront examinées dans le cadre du projet Gen-lex, que le Conseil fédéral soumettra aux Chambres dans le courant de l'année 2000.

Source: DFI/DETEC, "Plus de sécurité dans l'utilisation des OGM», Communiqué de presse, 25.8.99.

\section{Résultats de la conférence extraordinaire}

Lorsqu'il est devenu apparent qu'aucun compromis n'était possible entre le «groupe de Miami» et les autres Etats, les travaux d'élaboration du Protocole sur la biosécurité ont été suspendus. Les délégués ont convenu de reprendre les pourparlers au plus tard lors de la $5^{\mathrm{e}}$ Conférence des parties, qui se tiendra en principe en mai 2000. Malgré l'échec des négociations, il a été décidé d'intituler le document Protocole de Cartagena. Dans un communiqué de presse $^{37}$, la Suisse a fait part de sa déception après l'échec des négociations, alors qu'elle avait pris l'initiative de créer un groupe de compromis composé de pays défendant une position médiane afin de trouver une base d'accord minimum.

Comme le soulignent certains observateurs ${ }^{38}$, d'aucuns estiment que les OVM sont potentiellement dangereux, à la fois pour l'environnement et pour la santé humaine, mais d'autres y voient des possibilités d'une amélioration miraculeuse des récoltes et des médicaments. Rapprocher ces points de vue nécessitera un

36. WWF Schweiz, «Offizielle Schweiz missachtet Anliegen des Südens», Pressemitteilung, 16.2.99.

37. DETEC, «Négociations suspendues, la Suisse déçue», Communiqué de presse, 24.2.99.

38. Bulletin des négociations de la Terre, vol. $9, \mathrm{n}^{\circ} 117$. 
consensus scientifique plus clair, la sensibilisation du public aux risques encourus et une meilleure compréhension des implications écologiques, économiques et sociales de la biotechnologie.

\section{Commissions consultatives pour les questions ayant trait au génie génétique}

II existe en Suisse deux commissions consultatives, nommées par le Conseil fédéral, chargées des questions ayant trait au génie génétique.

• La Commission fédérale pour la sécurité biologique (CFSB) a été instituée en 1997. Elle sert de forum dans lequel se tient le débat scientifique sur le génie génétique au sein de la Confédération. Elle se compose d'experts issus de l'industrie, de l'agriculture et de la recherche, ainsi que de représentants des organisations de l'environnement et de défense des consommateurs. Durant ses deux premières années de fonctionnement, la commission a pris position sur tous les projets législatifs se rapportant au génie génétique (trois nouvelles ordonnances sur les OGM, projet Gen-lex). Elle a en outre pris position sur les disséminations expérimentales d'OGM et sur l'autorisation d'utiliser, en Suisse, du maïs transgénique comme denrée alimentaire ou fourrage.

Le premier rapport d'activité de la commission a été rendu public en août 1999 et peut être consulté sur Internet (www.uvek.admin.ch/doku/presse/1999/f).

Personne de contact pour la commission: Karoline Dorsch-Häsler (OFEFP), secrétaire générale de la commission, tél. 031/323.03.55.

- La Commission fédérale d'éthique pour le génie génétique dans le domaine non humain a été nommée par le Conseil fédéral fin avril 1998, à la veille de la votation sur l'initiative pour la protection génétique. La promptitude avec laquelle le Conseil fédéral avait nommé les 12 membres de la commission avait soulevé de nombreuses critiques parmi les initiants, qui voyaient là une stratégie pour rassurer la population et affaiblir l'initiative. Depuis lors, la commission a commencé ses travaux et défini ses objectifs. Cet organe consultatif a tout d'abord pour but de transmettre ses recommandations au Conseil fédéral et au Parlement en matière de biotechnologie et de génie génétique dans les domaines non humains. Elle devra également prendre position sur les nouvelles évolutions et les projets de recherche (en laboratoire comme au champ) dans ce domaine. Enfin, la commission est chargée d'encourager une large discussion publique sur les questions liées à l'application du génie génétique aux animaux et aux plantes.

La plupart des membres de la commission sont issus du milieu académique suisse, avec une prédominance de théologiens et de philosophes. Après avoir refusé sa nomination, Florianne Koechlin, opposante connue aux nouvelles biotechnologies, a finalement accepté d'y siéger.

Personne de contact pour la commission: Ariane Willemsen (OFEFP), secrétaire générale de la commission, tél. 031/323.83.83.

Sources:

DETEC, "La Commission fédérale pour la sécurité biologique présente son rapport", Communiqué de presse, 18.8.99.

"Génie génétique, une éthique pour la pratique", Vision. Le magazine suisse de la science et de l'innovation, 1/99.

\section{SOURCES}

Bulletin des négociations de la Terre, vol. 9, n 117, février 1999.

CICDD, Passerelles, «Prévention des risques biotechnologiques: incompatibilité entre la protection de la biodiversité et les règles de l'OMC? », vol. $1, \mathrm{n}^{\circ} 0$, novembre 1998; «Des considérations relatives aux impacts sur le commerce bloquent le protocole sur la prévention des risques biotechnologiques", vol. 1, n 2, mars-avril 1999.

Greenpeace International, Commentaires sur l'ébauche du texte de négociation (Protocole sur la biosécurité), février 1999.

SITES INTERNET

Convention sur la biodiversité: www.biodiv.org.

Bulletin des négociations de la Terre: www.iisd.ca/french/index.html. 


\subsection{MISE EN FUVRE DE LA CONVENTION DE LUTTE CONTRE LA DÉSERTIFICATION}

Les années de sécheresses de 1970-73 en Afrique, qui ont aggravé le phénomène de la désertification, ont donné naissance à un vaste mouvement de prise de conscience de la gravité du problème. Aussi, à la demande des pays africains, la communauté internationale a reconnu, lors de la Conférence de Rio en 1992, la nécessité de mettre en place, à côté de la Convention-cadre sur les changements climatiques et de la Convention sur la diversité biologique, une convention internationale de lutte contre la désertification. Comptant aujourd'hui 159 Etats parties, cet instrument juridique, adopté en 1994 et entré en vigueur en décembre 1996, témoigne de la préoccupation de la communauté internationale pour ce problème reconnu d'envergure mondiale. La Suisse, qui a déjà joué un rôle particulièrement important lors de l'élaboration de cette convention, en soutient à présent la mise en œuvre.

Menaçant environ le quart de la portion terrestre de notre planète et touchant directement un peu plus de 250 millions de personnes, la désertification, qui constitue un véritable obstacle au développement durable, apparaît comme une forme particulièrement dévastatrice de la dégradation de l'environnement, notamment en milieu aride et semi-aride. De par l'importance de ses conséquences, la désertification s'apparente à certains autres phénomènes environnementaux globaux. L'Afrique, dont $73 \%$ des terres arides sont déjà touchées par la désertification, n'est pas le seul continent où s'étend ce phénomène. La désertification constitue, sur tous les continents, une menace sérieuse qui touche, plus ou moins sévèrement, 110 pays et près de $70 \%$ des terres agricoles arides dans le monde.

Contrairement à ce que l'on croyait, le phénomène de la désertification n'est pas dû uniquement aux sécheresses, mais est causé, comme le dit le texte de la convention, «par des interactions complexes entre facteurs physiques, biologiques, politiques, sociaux, culturels et économiques». Par la baisse des rendements agricoles et la diminution, voire la destruction, de la couverture végétale qu'elle entraîne, la désertification est un facteur important de malnutrition, de famine, de problèmes de santé, de désorganisation sociale et, en fin de compte, d'appauvrissement. Mais elle se révèle aussi comme une conséquence de la pauvreté, ce qui fait d'elle un chaînon important d'un cercle vicieux particulièrement funeste.

\section{$\square$ Origines et principes de la Convention de lutte contre la désertification}

L'élaboration d'une Convention de lutte contre la désertification (CCD, Convention to Combat Desertification) a été décidée par la communauté internationale en juin 1992, au cours du Sommet de la Terre à Rio de Janeiro. Ainsi, peu après, le 22 décembre 1992, par la résolution 47/188, la 47e Assemblée générale de l'Organisation des Nations Unies (ONU) a institué un comité intergouvernemental chargé de négocier et d'élaborer, sur la base des directives d'Action 21, une convention internationale de lutte contre la désertification. Après des tractations ardues, ladite convention a été adoptée et signée à Paris respectivement les 17 juin et 14 octobre 1994. Elle est entrée en vigueur le 26 décembre 1996, après avoir recueilli les 50 ratifications nécessaires à cet effet.

Cette convention est donc un texte de droit international ayant force obligatoire pour tous les pays qui la ratifient ou y adhèrent. Son originalité s'exprime essen- 
tiellement par le fait qu'elle aborde le phénomène de dégradation des sols de manière globale, se démarquant par là des approches sectorielles antérieures. Elle s'appuie sur une série de principes novateurs. C'est ainsi qu'elle est la première convention internationale à consacrer la nécessité d'une participation effective à tous les niveaux (local, national et régional) des populations locales, des organisations non gouvernementales, des utilisateurs des ressources, sans oublier les femmes et les jeunes auxquels elle accorde une place très importante. S'agissant des ONG, il convient de relever que, suivant l'esprit de Rio, elles ont pu, grâce à l'accréditation de plus de 300 d'entre elles auprès du Comité international de négociation de la convention, jouer un rôle non négligeable dans la formulation de celle-ci. Un autre principe déterminant de ce document encourage des relations de partenariat entre les divers acteurs, à savoir les populations, les collectivités locales, les ONG, les différentes organisations bilatérales ou multilatérales et les services étatiques, et vise à tous les responsabiliser par rapport aux actions prévues à travers des engagements pris dans le cadre d'accords de partenariat. Enfin, la convention met aussi l'accent sur la nécessité d'une lutte intégrée et la création d'un environnement favorable, et mise pour cela sur des programmes d'action très larges s'inscrivant dans le cadre d'un développement durable à long terme.

La mise en œuvre de la convention repose sur des engagements pris par les pays parties développés et par les pays parties touchés. Relevons notamment les points suivants ${ }^{39}$ :

• Les pays parties développés s'engagent

- à appuyer activement l'action menée par les pays en développement touchés, en particulier ceux qui, parmi eux, se trouvent en Afrique et sont les moins avancés;

- à fournir des ressources financières importantes ainsi que d'autres formes d'appui aux fins de la lutte contre la désertification, et à favoriser la mobilisation de fonds nouveaux et additionnels;

- à faciliter l'accès des pays touchés à des technologies, connaissances et savoir-faire écologiquement appropriés;

- à renforcer les capacités propres des pays en développement touchés.

๑ Les pays parties touchés s'engagent de leur côté

- à accorder la priorité voulue à la lutte contre la désertification et à mobiliser des ressources financières nationales nouvelles et supplémentaires;

- à établir des stratégies et des priorités pour mener cette lutte;

- à s'attaquer aux causes profondes de la désertification et donc à accorder une attention particulière aux facteurs socio-économiques qui contribuent à la désertification;

- à sensibiliser les populations locales, en particulier les femmes et les jeunes, et à faciliter leur participation, avec l'appui des ONG, à l'action menée;

- à créer un environnement porteur, notamment en renforçant la législation pertinente, en intensifiant les réformes engagées en matière de décentralisation, et en élaborant de nouvelles politiques à long terme et de nouveaux programmes d'action;

- à coopérer entre eux pour lutter contre la désertification. 
La conférence des parties, qui constitue l'organe suprême de la CCD, doit permettre de faire régulièrement le point, lorsqu'elle se réunit, sur l'évolution de la mise en œuvre de la convention. Depuis l'entrée en vigueur de cette dernière, trois conférences des parties ont eu lieu. Le Secrétariat de la convention, qui jusqu'en décembre 1998 était installé à Genève et qui s'est déplacé depuis à Bonn, a pour fonction de coordonner, en le stimulant, le processus de mise en œuvre de la convention et d'organiser les conférences des parties.

\section{$\square$ La $1^{\text {re }}$ Conférence des parties (COP-1)}

C'est au siège de l'Organisation pour l'alimentation et l'agriculture (FAO), à Rome, que se sont réunis, du 29 septembre au 10 octobre 1997, plus de 1100 délégués venant de 102 Etats parties, de 18 Etats observateurs, de 15 organismes intergouvernementaux et de 62 organisations non gouvernementales, pour prendre part à la $1^{\text {re }}$ Conférence des parties. Lors de cette session, plusieurs décisions d'importance ont été prises concernant les fonctions du Mécanisme mondial, le choix d'une structure d'accueil pour ce mécanisme (Fonds international de développement agricole - FIDA), ainsi que le siège du Secrétariat permanent (Bonn/Allemagne). En ce qui concerne le Mécanisme mondial, il s'agit d'une institution propre à la Convention de lutte contre la désertification qui n'est en soi pas un fonds, mais est seulement destinée à mobiliser et à canaliser des ressources financières en vue de la mise en œuvre de la CCD. Ont également été arrêtés un budget pour les différents organes de la convention, ainsi qu'un règlement intérieur et des règles de gestion financière. En outre, les délégués des Etats parties ont discuté de la question de la collaboration entre les différentes conventions environnementales, tout particulièrement celles de Rio, et d'une participation du Fonds pour l'environnement mondial (FEM) au financement des actions de lutte contre la désertification. En effet, si le FEM a été conçu comme un instrument de financement au service des conventions environnementales, il ne comporte cependant pas de guichet spécial en faveur de la lutte contre la désertification. Quant au compromis stipulant que le FEM peut maintenant financer des actions de lutte contre la désertification dans la mesure où celles-ci servent également les buts des autres conventions (notamment sur les changements climatiques et sur la diversité biologique), il ne répond pas aux attentes des pays touchés, lesquels continuent donc à réclamer que la $\mathrm{CCD}$ bénéficie des mêmes prestations de la part du FEM que les conventions pour lesquelles celui-ci a été créé.

Simultanément, les 2 et 3 octobre, s'est aussi réuni le Comité de la science et de la technologie (CST), un organe subsidiaire de la conférence des parties chargé d'assister cette dernière en lui apportant les informations scientifiques et techniques dont elle a besoin pour la lutte contre la désertification et la sécheresse. Ce comité a élaboré et adopté un programme de mise en réseaux des institutions spécialisées s'occupant du problème de la dégradation des terres et a approfondi la question de la définition d'indicateurs d'impact des mesures de lutte contre la désertification. De plus, il a été décidé de charger le CST d'examiner, en vue de sa deuxième réunion, si et, le cas échéant, comment les connaissances et savoirs traditionnels pourraient être valorisés, parallèlement à la technologie moderne, dans le cadre de la lutte contre la désertification.

Enfin, la COP-1 a convenu de faire désormais une place dans le programme des futures conférences à un dialogue entre les ONG et les Etats parties. Notons 
encore que, parallèlement à cette COP-1, se sont déroulées d'autres manifestations, à savoir notamment un forum des $\mathrm{ONG}$, un forum international des maires et un séminaire pour les médias sur divers thèmes choisis.

\section{$\square$ La $2^{e}$ Conférence des parties}

Du 30 novembre au 11 décembre 1998 s'est tenue à Dakar la $2^{\mathrm{e}}$ Conférence des parties (COP-2). Cette fois-ci, ce sont plus de 500 représentants de 132 pays qui l'ont suivie. La délégation suisse était conduite par Henri-Philippe Cart, vicedirecteur de la DDC. A nouveau, ce sont essentiellement des discussions sur des préalables aux mesures effectives de lutte contre la désertification, à savoir des questions d'organisation et d'ordre institutionnel, qui ont animé les débats. A relever l'invitation faite aux pays d'Europe centrale et de l'Est à soumettre, lors de la COP-3, une proposition d'annexe traitant de la mise en œuvre de la convention dans cette région. En ce qui concerne les mesures prises par les pays affectés, on retiendra que la COP-2 a été l'occasion, pour tous ceux d'entre eux qui le désiraient, de faire un premier compte-rendu oral de leurs efforts.

Le Comité de la science et de la technologie, qui s'est réuni en marge de la COP2 , du $1^{\text {er }}$ au 4 décembre, a aussi pris des décisions importantes. Cependant, on constate que, là non plus, tous les préalables à l'action concrète ne sont pas encore levés. Ainsi, alors que la constitution d'un groupe spécial chargé de suivre les débats sur le recours aux connaissances techniques et traditionnelles était décidée, l'adoption d'un accord entre la COP et le FIDA concernant le Mécanisme mondial, ainsi que la proposition d'établir un comité chargé de vérifier l'application de la convention ont bien été discutées, mais finalement reportées à la COP-3. Néanmoins, les délégués se sont montrés satisfaits des débats sur les connaissances et les savoirs traditionnels ainsi que sur la mise en œuvre expérimentale des plans d'action nationaux. Ils ont aussi apprécié de pouvoir traiter de ces thèmes avec les ONG. Pourtant, il faut noter que ces réflexions sont demeurées très générales et abstraites. Pour terminer, les discussions qui étaient prévues depuis la COP-1 sur l'évaluation des réseaux existants ainsi que sur la mise en œuvre du Mécanisme mondial, qui sera cogéré par le FIDA, la Banque mondiale et le PNUD, n'ont pas pu être menées à terme.

\section{$\square$ La $3^{e}$ Conférence des parties}

La $3^{\mathrm{e}}$ Conférence des parties (COP-3) s'est déroulée à Recife, au Brésil, du 15 au 26 novembre 1999. Parallèlement, du 16 au 19 novembre, s'est réuni le Comité de la science et de la technologie. Si un grand nombre de parlementaires étaient présents lors de ces réunions, l'absence de participation à haut niveau, essentiellement de pays de l'OCDE, a été vivement critiquée et a renforcé l'opinion selon laquelle les pays industrialisés n'éprouvent que peu d'intérêt pour la CCD. La délégation de la Suisse était à nouveau conduite par le vice-directeur de la DDC, Henri-Philippe Cart.

C'est essentiellement un sentiment général de déception qui est ressorti de cette réunion. Les espoirs placés dans un examen des programmes nationaux et régionaux qui aurait permis d'identifier les meilleures pratiques et d'échanger des expériences furent largement déçus: si $80 \%$ des pays africains parties chargés de présenter leur rapport à la COP-3 ont rempli leurs engagements, le débat est resté, une fois de plus, centré sur les questions concernant la structure de la 
convention, ses procédures et les mécanismes d'évaluation de sa mise en application, ainsi que sur le rôle du Secrétariat. La décision de réviser la stratégie à moyen terme de celui-ci a obligé le secrétaire exécutif à établir des priorités dans son programme d'activités, notamment celle de faciliter l'évaluation de la mise en application de la convention. C'est aussi dans une atmosphère tendue que se sont déroulées au cours de cette conférence les longues et difficiles négociations concernant le budget. Le secrétaire exécutif s'est vu accorder finalement l'autorisation d'opérer des transferts entre les principales lignes budgétaires mais avec, en contrepartie, l'obligation de rendre compte à la COP-4 de l'exécution du budget et de son efficience.

Bien que cette conférence ait été largement critiquée, il faut noter plusieurs décisions importantes: l'approbation de l'accord entre le FIDA et la COP concernant le Mécanisme mondial, renvoyée lors de la COP-2; la décision d'encourager les pays d'Europe centrale et de l'Est à poursuivre leurs consultations autour du projet d'annexe pour qu'il puisse être adopté lors de la COP-4 (prévue provisoirement en octobre 2000 à Bonn) ; l'adoption de l'Initiative de Recife, qui appelle les parties à revoir leurs engagements à l'égard du processus de la CCD, à travers essentiellement la législation, les politiques éducationnelles et culturelles ainsi que la promotion de la société civile, des communautés locales ainsi que des jeunes et des femmes; a également été réaffirmée la nécessité d'une collaboration étroite avec les instances compétentes des autres conventions de Rio. Pour le reste, la COP s'est bornée à confirmer le rôle important attribué aux ONG dans la mise en œuvre de la convention.

\section{$\square$ Position de la Suisse par rapport à la CCD}

La Suisse a activement participé à l'élaboration, puis aux premières étapes de la mise en ouvre de cette convention et a fourni aussi une contribution importante en tant que pays hôte du Secrétariat intérimaire, notamment par les prestations en nature et d'ordre financier que cela implique (mise à disposition gratuite de locaux, prise en charge de certains frais de fonctionnement du Secrétariat, etc.). Sa ratification, en janvier 1996, lui a permis de confirmer, sur un plan international, son engagement en faveur de la convention. Mis à part une large contribution à des actions permettant de lutter contre la désertification, la Suisse, dans ses projets de coopération bilatérale, favorise une collaboration étroite entre les acteurs de même que le renforcement des capacités des populations. De plus, elle s'efforce d'assurer l'établissement de relations de partenariat entre les communautés de base et les autres acteurs à tous les stades de développement des projets. Enfin, la Suisse, qui encourage toujours davantage les ONG du Sud et du Nord à prendre une part active aux conférences internationales, a fortement soutenu la participation des ONG du Sud aux travaux, tant pendant la période de négociation de la convention qu'à l'occasion des réunions de la conférence des parties.

ASTM 1996, «Convention de lutte contre la désertification», pp. 57-59.

\section{SOURCES}

ONU, Convention des Nations Unies sur la lutte contre la désertification (UNCCD), ONU, Genève, 1997.

ONU, Cap sur terre, ONU, Genève, 1998.

SITE INTERNET

Site officiel de la Convention de lutte contre la désertification: www.unced.de.

ADRESSE UTILE

Secrétariat de la Convention de lutte contre la désertification, Haus Carstanjen, Martin Luther King Strasse 8, 53175 Bonn, Allemagne, tél. 0049/228.815.28.00, fax 0049/228.815.28.98/99. 